\title{
The Citizen as a Key Point of the Policies: A First Approach to Auralization for the Acoustic Perception of Noise in an Urban Environment ${ }^{+}$
}

\author{
Rosa Ma Alsina-Pagès * (i) and Pau Bergadà \\ GTM-Grup de Recerca en Tecnologies Mèdia, c/Quatre Camins, 30, 08022 Barcelona, Spain; \\ pau.bergada@salle.url.edu \\ * Correspondence: rosamaria.alsina@salle.url.edu; Tel.: +34-932902455 \\ + Presented at the 5th International Electronic Conference on Sensors and Applications, 15-30 November 2018; \\ Available online: https: / / ecsa-5.sciforum.net.
}

Published: 14 November 2018

\begin{abstract}
The improvement of the quality of life in the framework of the smart city paradigm cannot be limited to a set of objective measures carried out over several critical parameters (e.g., noise, air pollution). The citizen's perception of the problem to be solved, as well as the perception of the improvement achieved with the policies defined for this purpose are more important than the objectivity and the measurement of the change achieved. A first auralization approach for the evaluation of the acoustic perception of street noise is presented in this work. The wireless acoustic sensor network can pick up street noise and can even record specific sounds that reach a higher equivalent level for study, but the most important thing for administration is whether the neighbor has noticed an improvement in the quality of life. This work is a first approximation to an estimation of the real perception of citizens of the street urban noises collected by a low-cost wireless acoustic sensor network.
\end{abstract}

Keywords: noise; auralization; citizen; smart city; wireless acoustic sensor network

\section{Introduction}

Because of population growth and the consequent expansion of transportation systems, including highways, railways, and airways, environmental noise pollution is increasing year after year. Noise pollution continues to constitute a major environmental health problem in Europe [1]. From all the health effects, annoyance is one of the most well-known effects of environmental noise [2]; however, it is not merely an annoyance, since several works point out health-related problems such as sleep disorders [3], learning impairment [4], and heart disease [5]. Most of the conducted studies address the effects of long-term exposure to environmental noise and are mainly focused on concentration, sleep disturbance, and stress [6] issues, emphasizing the especially negative effects on children [7].

The European Union reacted to this alarming increase of environmental noise pollution, especially in large population cities, approving the Environmental Noise Directive 2002/49/EC (END) [8]. In accordance with the END, the CNOSSOS-EU methodological framework pretends to improve the consistency and comparability of noise assessment results across the EU Member States [9] for its application. The main pillars of the END are the following: (i) determining the noise exposure; (ii) making the updated information related to noise available to citizens, and (iii) preventing and reducing the environmental noise where necessary.

Recent studies show that the effects of noise on people not only depend on the level of noise, but also on the type of sound. In fact, in 2018, the WHO has incorporated into its study noises such as leisure noise and wind turbine noise (http:/ / www.euro.who.int/en/publications/abstracts/environmental- 
noise-guidelines-for-the-european-region-2018). This principle is the basis for the work carried out by this team within the framework of the European project LIFEDYNAMAP [10]. The ANED [11], the anomalous noise event detector, has been designed to rule out non-traffic noise events; this algorithm separates abnormal noises from the road traffic noise. The ANED is an algorithm based on the spectral distribution of the different types of noise to identify them properly, and throughout the study time, it has been proven that depending on the propagation of sound, the identification conditions may change [12]. Furthermore, by changing the temporal spectrum distribution of the signal, human perception may also change [13]. This work intends to be a first step in the framework of the concept of auralization in an urban environment [14] with non-traffic-related noise (anomalous noise events (ANE)), to evaluate a first observation of the effect of the channel [15] on the spectrum-temporal vision of the real-operation signal collected in the Milan pilot project DYNAMAP [10]. The final goal of this preliminary study is two-fold: on the one hand, have a first glance at the possible influence of the channel propagation on the accuracy of the ANED and, on the other hand, the possibility of the change in the annoyance of the neighborhood depending on the impulse response of the propagation of the noise channel.

This paper is structured as follows. In Section 2, brief details of the LIFE-DYNAMAP project are given. In Section 3 the mathematical models used for propagation are detailed, the results of which are presented in Section 4. Finally, several conclusions are described in Section 5, and future work is proposed.

\section{The DYNAMAP Project and Real-Operation Recordings}

In this framework, the DYNAMAP project [10] aims to deploy a low-cost hybrid WASN to tailored noise maps representing the acoustic impact of road infrastructures in real time, using a Geographic Information System (GIS) platform. The project includes the deployment of two pilot areas in Italy, the A90 motorway in Rome (for the suburban scenario) and District 9 in Milano (urban area). The system has to operate $24 \mathrm{~h}$ a day, 7 days a week. In order to monitor the impact of the road infrastructures solely, the events that are unrelated to road traffic noise, denoted as ANE, should be removed from the noise map generation [16] to avoid its impact.

In District 9 in Milan there are currently 24 low-cost, high-capacity sensors deployed in a WASN. We have collected ANE data from two sensors (hb137 and hb145), the performance of which is the closest to open air due to the fact that they are located near parks, and not in narrow streets. For more details about the location of the sensors, the reader is referred to [17]. The data were recorded during two complete days - one weekday, on Thursday, and one weekend day, on Sunday-gathering $20 \mathrm{~min}$ of audio data each hour, in order to maximize the diversity of the recorded ANEs. For the acoustic data gathering, Bluewave, the partner of the DYNAMAP project that handles sensors' hardware design and maintenance, provided us access to the recorded data files in the cloud, which were subsequently downloaded. The next step was labeling by subjective listening to half of the available audio (all odd hours of the $20 \mathrm{~min}$ recorded: $1 \mathrm{~h}, 3 \mathrm{~h}, 5 \mathrm{~h}, \ldots, 23 \mathrm{~h}$ ), which was performed by five trained listeners. From those labeled events, we collected several significant noises (airplane, bell, and horn) to conduct this first stage of the study.

\section{Outdoor Propagation Models}

In this work, we considers the sound signal radiating isotropically as a spherical wave-front [18]. In such a case, the free-field intensity of the radiation reduces with the inverse square of the distance. If we take into account the sound pressure $(P)$, this relationship translates into the following relationship:

$$
P(R)=\frac{P(E)}{r},
$$


where $R$ is the location of the receiver, $E$ is the location of the emitter, and $r$ is the euclidean distance between both. In this work, we do not consider high frequency attenuation due to atmospheric scattering.

Regarding sound reflection models, we assume pure specular reflectors with obstacles much bigger than the emitted sound wavelength since it is an urban scenario. We also take into account two channel models in an urban scenario. The first one is a two taps channel (Channel A) where the emitter and receiver are separated $5 \mathrm{~m}$. In Channel A, we consider a direct path and a ground reflected path $(7 \mathrm{~m}$ long). However, we have designed a more challenging channel with a direct path $(8 \mathrm{~m}$ long), a ground-reflected path (10 m long), and two more paths reflected or refracted by nearby walls and/or vegetation (14 m and $16 \mathrm{~m}$ long). Each tap introduces an attenuation, which is, as stated above, inversely proportional to the length of the path. The phase $(\theta)$ of each path is uniformly distributed between 0 and $2 \pi$. Then, the impulse response of the channel can be expressed as:

$$
h(n)=\sum_{n=0}^{N-1} \frac{1}{r_{n}} e^{-j \theta}
$$

where $r_{n}$ is the attenuation of path $n$ and $N$ is the number of paths of the channel ( $N=2$ for Channel A, and $N=4$ for Channel B).

\section{Results}

In this section, we evaluate the changes suffered in the frequency domain when recorded ANEs were propagated through two different multipath channels (Channel A and Channel B, explained in Section 3). We show in Figures 1-3 the outcomes related to different ANEs, i.e., the noise of an airplane, a bell, and a horn. For the sake of brevity, we only show these three examples, which are representative of the phenomena we want to outline. For each of them, we show the spectrogram of the emitted signal in the upper plot, the spectrogram of the received signal through Channel A in the mid-left plot, and the spectrogram of the received signal through Channel B in the mid-right plot. Finally, in the lower plots of each figure, we show the accumulated energy at the receiver when propagating through Channels A and B, on the left- and right-hand side, respectively. The spectrogram chops the signal into 40-ms segments, which were windowed with a Hanning window to reduce leakage and transformed into the frequency domain by means of a 2048-point FFT, displayed in a natural scale. Consecutive segments were overlapped by a factor of $87.5 \%$ to maximize the probability of detection.

In Figure 2, we can observe two phenomena. The first one is that the frequency distribution of the energy changed depending on the type of channel, and the second one is that the intensity of the received sound may depend on the number of paths of the channel and the phase of each of them when impacting the receiver. In Figure 3, we can observe that Channel A and Channel B influenced the intensity of the high frequency components (e.g., the component at $10 \mathrm{kHz}$ ), as well as their time length. In Figure 1, we can observe that the intensity and number of high frequency components were both reduced in Channel B compared to Channel A.

In all of the figures, we can observe that the variation of the accumulated energy at the emitter point was more similar to that at the receiver point when propagating through a low number of tap channel (i.e., Channel A), rather than through a higher number of tap channel (i.e., Channel B). The fact of having a higher number of replicas with random phase added together increased the probability of having the maximums of energy at different time instants. 

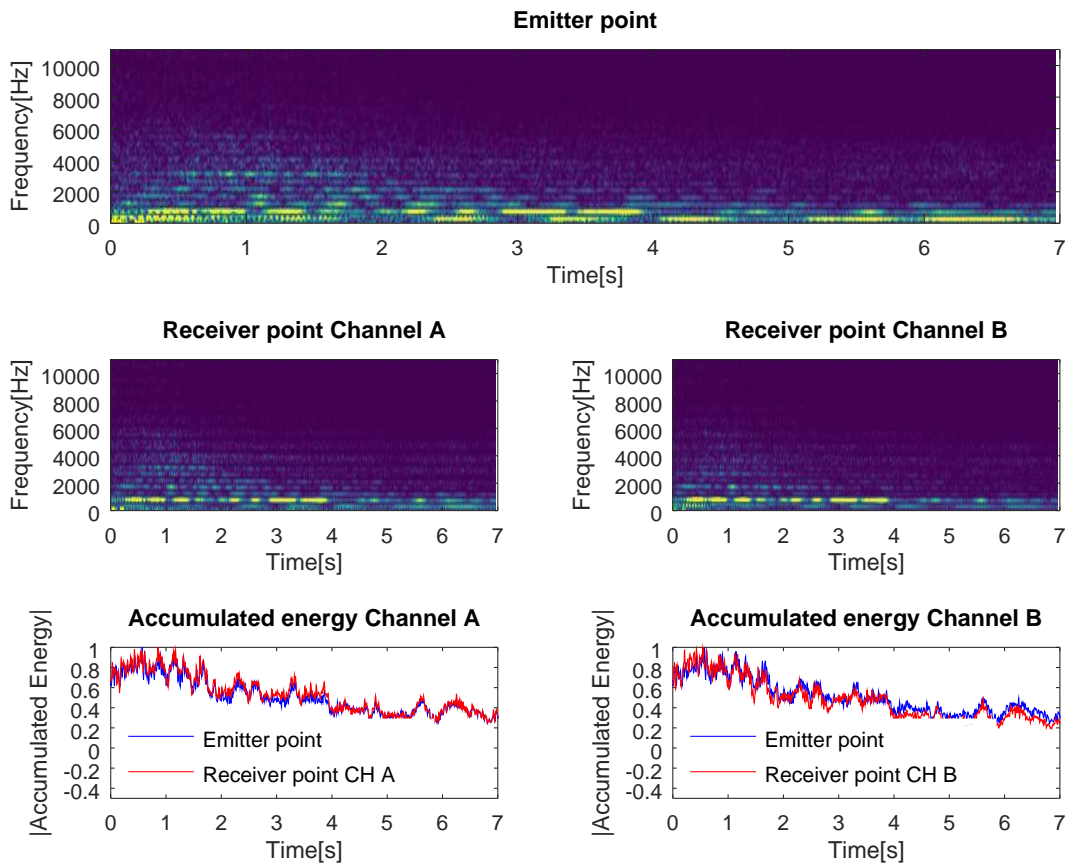

Figure 1. Anomalous noise event labeled as an airplane. The spectrogram of the emitted signal in the upper plot; in the mid-left plot, the spectrogram of the received signal through Channel A; in the mid-right plot, the spectrogram of the received signal through Channel B; in the lower-left plot, the accumulated energy through Channel A; and in the lower-right plot, the accumulated energy through Channel B.
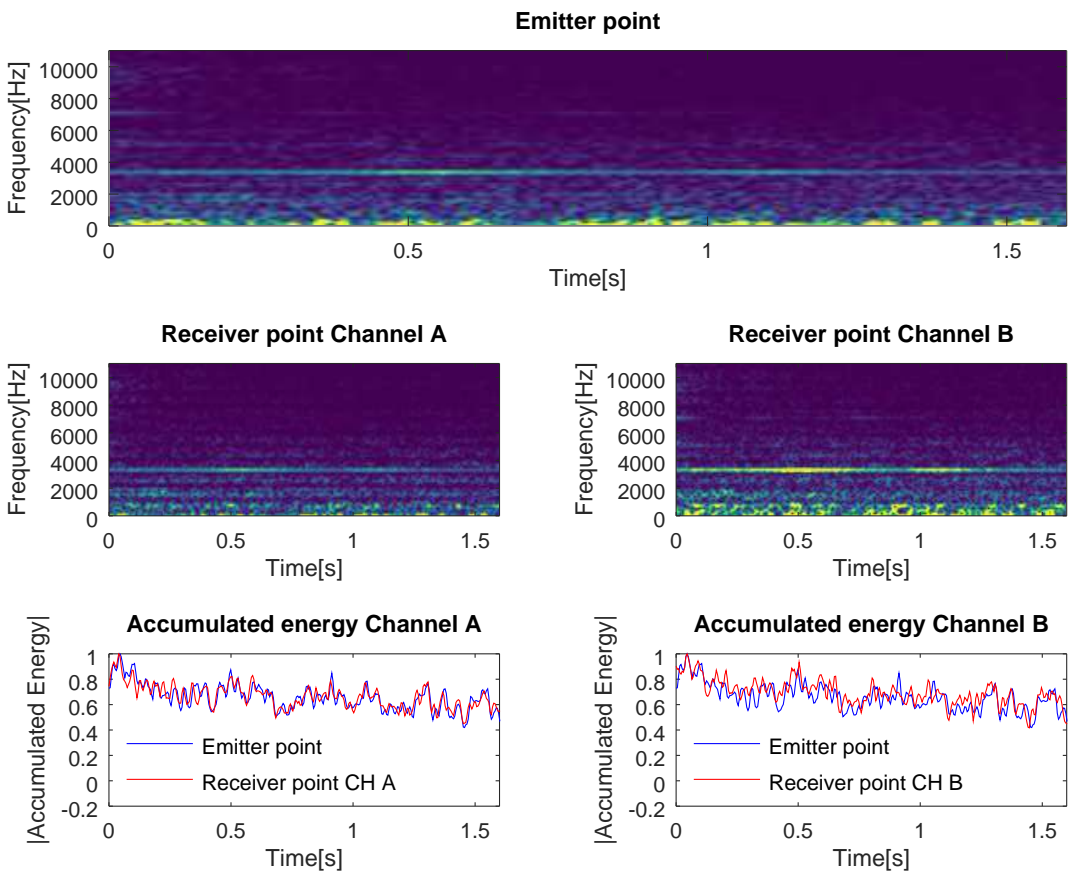

Figure 2. Anomalous noise event labeled as a bell. The spectrogram of the emitted signal in the upper plot; in the mid-left plot, the spectrogram of the received signal through Channel A; in the mid-right plot, the spectrogram of the received signal through Channel B; in the lower-left plot, the accumulated energy through Channel A; and in the lower-right plot, the accumulated energy through Channel B. 

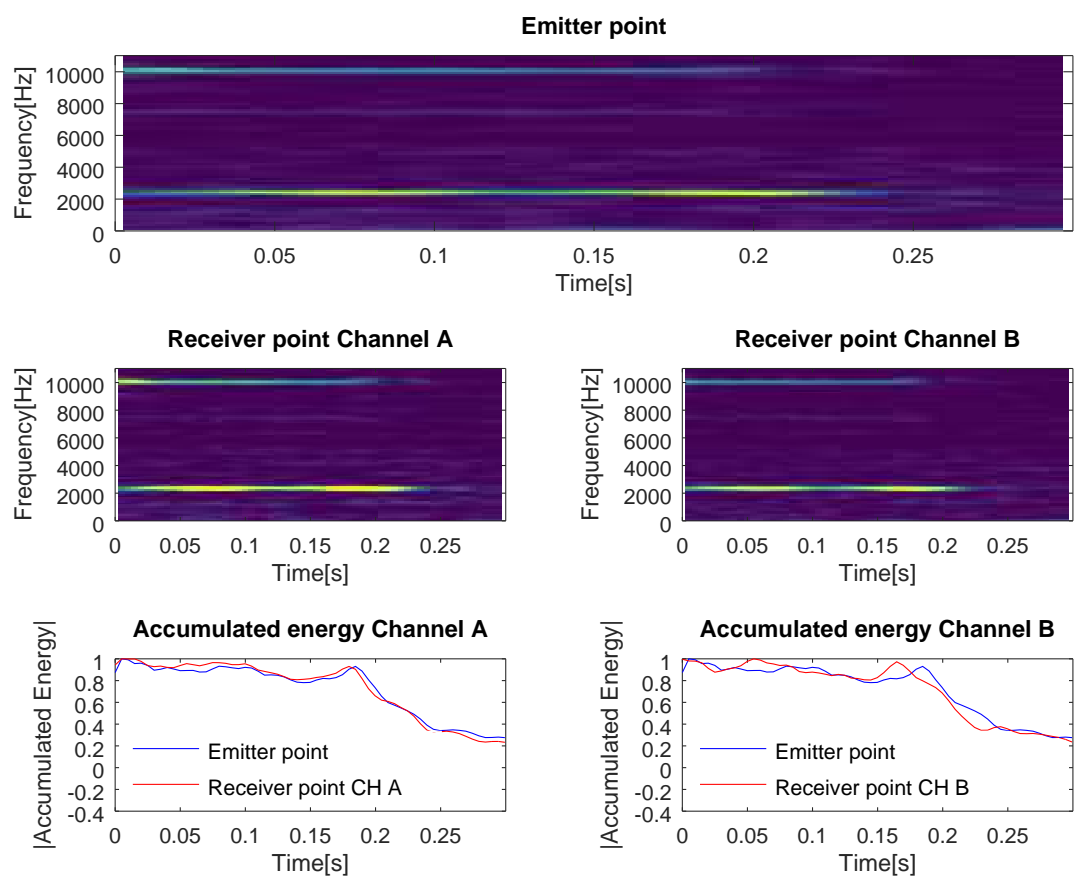

Figure 3. Anomalous noise event labeled as a horn. The spectrogram of the emitted signal in the upper plot; in the mid-left plot, the spectrogram of the received signal through Channel A; in the mid-right plot, the spectrogram of the received signal through Channel B; in the lower-left plot, the accumulated energy through Channel A; and in the lower-right plot, the accumulated energy through Channel B.

\section{Conclusions}

The work presented in this paper is a preliminary study to determine the spectro-temporal variations of acoustic signals in the presence of different types of propagation channels in an urban environment. On the one hand, the qualitative evaluations developed in this work present substantial variations both in terms of spectral distribution energy and in temporal variations due to delay. These variations can have severe effects on the detection of anomalous events using the ANED algorithm.

On the other hand, it should be also taken into account whether these spectral-temporal variations have any effect on people living in the environment: Do these variations make the noises more annoying? Does perception change when the coefficients of the spectral and temporal energy distribution are modified?

The future lines of this work are going to focus on the quantification of spectro-temporal variations depending on the type of channel with which we are working. At the same time, the study will be generalized for all the ANE available in the project, and the degree of the detectionwill be determined as ANED accuracy for different types of channels. Finally, it is intended to study the degree of generalization of the detection of acoustic events in varying propagation environments, taking the example of a narrow street with tall buildings to a point surrounded by a park, much closer to what could be considered open air.

Author Contributions: R.M.A.-P. conceived of the experiments and wrote a part of the paper. P.B. coded the tests and wrote the rest of the paper.

Acknowledgments: The research presented in this work has been partially supported by the LIFE DYNAMAP project (LIFE13 ENV/IT/001254).

Conflicts of Interest: The authors declare no conflict of interest. 


\section{References}

1. European Commission. Report from the Commission to the European Parliament and the Council On the Implementation of the Environmental Noise Directive in accordance with Article 11 of Directive 2002/49/EC; Publications Office of the European Union: Brussels, Belgium, 2017.

2. Miedema, H.; Oudshoorn, C. Annoyance from transportation noise: relationships with exposure metrics DNL and DENL and their confidence intervals. Environ. Health Perspect. 2001, 109, 409-416.

3. Muzet, A. Environmental noise, sleep and health. Sleep Med. Rev. 2007, 11, 135-142.

4. Hygge, S.; Evans, G.W.; Bullinger, M. A prospective study of some effects of aircraft noise on cognitive performance in schoolchildren. Psychol. Sci. 2002, 13, 469-474.

5. Dratva, J.; Phuleria, H.C.; Foraster, M.; Gaspoz, J.M.; Keidel, D.; Künzli, N.; Liu, L.J.S.; Pons, M.; Zemp, E.; Gerbase, M.W.; et al. Transportation noise and blood pressure in a population-based sample of adults. Environ. Health Perspect. 2012, 120, 50-55.

6. Babisch, W. Transportation noise and cardiovascular risk. Noise Health 2008, 10, 27-33.

7. Regecová, V.; Kellerová, E. Effects of urban noise pollution on blood pressure and heart rate in preschool children. J. Hypertens. 1995, 13, 405-412.

8. Union, E. Directive 2002/49/EC of the European Parliament and the Council of 25 June 2002 relating to the assessment and management of environmental noise. Off. J. Eur. Commun. 2002, 189, 12.

9. Kephalopoulos, S.; Paviotti, M.; Anfosso-Lédée, F. Common Noise Assessment Methods in Europe (CNOSSOS-EU); Report EUR 25379 EN; Publications Office of the European Union: Brussels, Belgium, 2002; pp. 1-180.

10. Sevillano, X.; Socoró, J.C.; Alías, F.; Bellucci, P.; Peruzzi, L.; Radaelli, S.; Coppi, P.; Nencini, L.; Cerniglia, A.; Bisceglie, A.; et al. DYNAMAP—Development of low cost sensors networks for real time noise mapping. Noise Mapp. 2016, 3, 172-189.

11. Socoró, J.C.; Alías, F.; Alsina-Pagès, R.M. An Anomalous Noise Events Detector for Dynamic Road Traffic Noise Mapping in Real-Life Urban and Suburban Environments. Sensors 2017, 17, 2323.

12. Alías, F.; Alsina-Pagčs, R.M.; Orga, F.; Socoró, J.C. Detection of Anomalous Noise Events for Real-Time Road-Traffic Noise Mapping: The Dynamap's project case study. Noise Mapp. 2018, 5, 71-85.

13. Nilsson, M.; Forssén, J.; Lundén, P.; Peplow, A.; Hellström, B. LISTEN Auralization of Urban Soundscapes; Technical Report, Final Report to the Knowledge Foundation; Stockholm University: Stockholm, Sweden, 2011.

14. Hewett, D.P. Sound Propagation in an Urban Environment. Ph.D. Thesis, Oxford University, Oxford, UK, 2010.

15. Attenborough, K.; Li, K.M.; Horoshenkov, K. Predicting Outdoor Sound; CRC Press: Boca Raton, FL, USA, 2014.

16. Orga, F.; Alías, F.; Alsina-Pagès, R.M. On the Impact of Anomalous Noise Events on Road Traffic Noise Mapping in Urban and Suburban Environments. Int. J. Environ. Res. Public Health 2017, 15, 13.

17. Zambon, G.; Benocci, R.; Bisceglie, A.; Roman, H.E.; Bellucci, P. The LIFE DYNAMAP project: Towards a procedure for dynamic noise mapping in urban areas. Appl. Acoust. 2017, 124, 52-60.

18. Lamancusa, J. Noise Control, Outdoor Noise Propagation; Penn State University: Pennsylvania, PA, USA, 2000.

(C) 2018 by the authors. Licensee MDPI, Basel, Switzerland. This article is an open access article distributed under the terms and conditions of the Creative Commons Attribution (CC BY) license (http://creativecommons.org/licenses/by/4.0/). 